\title{
Testbench to characterize pixels of the Major Atmospheric Gamma-ray Imaging Cherenkov (MAGIC) telescope
}

\author{
F. Lucarelli* \\ M. Camara \\ P. Antoranz \\ Universidad Complutense de Madrid \\ Departmento Física Atómica \\ Molecular y Nuclear \\ Facultad de Ciencias Físicas \\ Madrid, Spain
}

\author{
J. M. Miranda \\ Universidad Complutense de Madrid \\ Departmento Física Aplicada 3 \\ Facultad de Ciencias Físicas \\ Madrid, Spain
}

\section{Asensio}

Universidad Politécnica de Madrid

Departmento Infraestructura

Sistemas Aeroespaciales y Aeropuertos

ETSIA

Madrid, Spain

\section{J. A. Barrio}

M. V. Fonseca

Universidad Complutense de Madrid

Departmento Física Atómica

Molecular y Nuclear

Facultad de Ciencias Físicas

Madrid, Spain

\begin{abstract}
Photomultipliers have a wide range of applications, from nuclear medicine to nuclear physics. In particular, they are commonly used in high energy physics and astrophysics. The Major Atmospheric Gamma-ray Imaging Cherenkov (MAGIC) telescope is an example of their applications. This work presents a test system for the characterization of the transient response of the photomultipliers used as fast light detectors in the telescope. The bias of the photomultiplier is described, taking into account its effects in the response to fast light pulses. Two methods are used to generate these pulses: 1. a specifically designed pulse generator, and 2. a plastic scintillator. For the former case, we also calculate the anode charge we expect in this setup to cross-check it with the actual measurements. () 2006 Society of Photo-Optical Instrumentation Engineers. [DOI: 10.1117/1.2336418]
\end{abstract}

Subject terms: astronomy; Cherenkov radiation; gamma-ray detectors; photomultiplier; transient response.

Paper 050700R received Aug. 27, 2005; revised manuscript received Jan. 2, 2006; accepted for publication Jan. 20, 2006; published online Aug. 31, 2006.

\section{Introduction}

The MAGIC telescope ${ }^{1}$ is an innovative detector to measure very high energy gamma radiation from astrophysical sources. The telescope detects the very short flashes of atmospheric Cherenkov radiation produced in the interaction of the cosmic gamma rays with the atmospheric nuclei. A shower of relativistic charged secondary particles develops deep in the atmosphere and produces a cone of Cherenkov radiation as their charged particles move downward faster than the speed of light in the air. ${ }^{2}$

The main and unique characteristic of the telescope is the huge diameter of its mirror, $17 \mathrm{~m}$. That, along with a system of signal transmissions based on optical fiber and digital sampling of signals with $300-\mathrm{MHz}$ Flash analogdigital converters (ADCs), allows for an extremely low energy threshold of primary gamma rays of $30 \mathrm{GeV}$.

The main detector, or camera, is located at the focus of

\footnotetext{
*Current affiliation: Università "La Sapienza," Dip. Physics, Ple. Aldo Moro 2, Roma 00185, Italy, E-mail: Fabrizio.Lucarelli@Roma1.infn.it 0091-3286/2006/\$22.00 @ 2006 SPIE
}

the telescope and consists of a matrix of 577 fast-response photomultipliers (PMTs), ${ }^{3}$ which register the very fast Cherenkov pulses (of 5- to 20-ns duration) emitted in the atmospheric cascades produced by the cosmic gamma rays. The camera, shown in Fig. 1, has a diameter of $1.5 \mathrm{~m}$, and is composed by an inner ring of 397 1-in. PMTs [model Electron Tubes (Middlesex, England) 9116B] and an outer ring of 180 1.5-in. PMTs (type ET9117B). All PMTs have a quantum efficiency of 25 to $30 \%$ in the spectral range 170 to $630 \mathrm{~nm} .{ }^{4,5}$ In a second phase of upgrading of the telescope, it is foreseen to use new hybrid light detectors (HPD) with quantum efficiency as high as $45 \% .^{6}$

In this work, we describe the design, construction, and tests of a testbench for the PMT ET9116. The PMT was equipped with a voltage divider suitable for rapid pulse mode of operation. Moreover, a high velocity pulse transmitter, which simulates the Cherenkov light to be detected by the telescope, was used to drive a fast UV LED, which was used as a light source for the PMT. ${ }^{7}$

This testbench was used to characterize the time response of the PMT ET9116B of the MAGIC camera, and can be used also to test other types of PMTs or different 


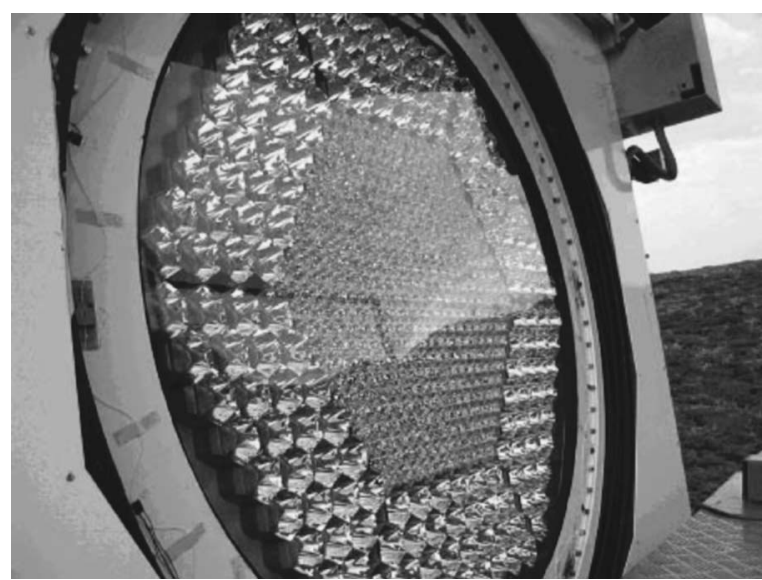

Fig. 1 The MAGIC telescope camera.

photodevices like HPDs or avalanche photodiodes (APDs). In particular, it has been used to calibrate and test the output signal transmission of a modified version of the standard MAGIC 1-in. PMT, which has been installed at the center of the camera to perform optical observations of variable astronomical objects. ${ }^{8,9}$

\section{Photomultiplier Polarization}

Figure 2 shows a scheme of the voltage divider chosen to polarize the PMT. This polarization network is suitable to operate the PMT in pulsed mode, where very brief and intense light pulses are to be detected. The anode $(a)$ is connected to the ground and the cathode $(k)$ is set to a high negative voltage. ${ }^{10}$ Setting the PMT gain to $10^{4}$, a voltage value $V_{h t}=1100 \mathrm{~V}$ has to be applied between the cathode and anode according to the specifications of the device.

The value of the resistor between the cathode and first dynode $(d 1)$ was chosen in such a way that the voltage $V_{k-d 1}$ was as high as possible but always below the permitted maximum voltage of $400 \mathrm{~V}$ reported in the PMT datasheets. That improves the time response of the PMT and avoids the appearance of "afterpulses." For the chosen resistor values, we get $V_{k-d 1}=360$ and $137 \mathrm{~V}$ between all other pairs of dynodes.

The three parallel-connected capacitors used in the last three stages serve as decoupling devices for the pulsed

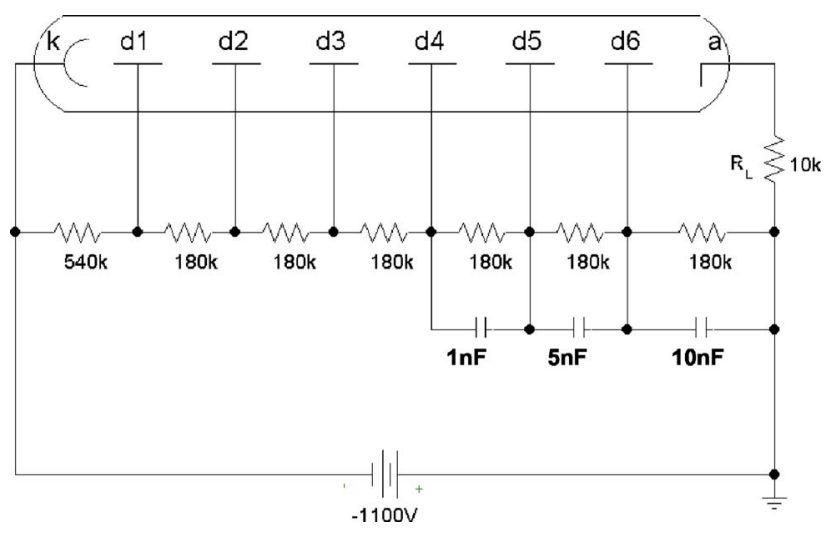

Fig. 2 Polarization network of the PMT. mode of operation. They supply the PMT tube with an electric charge all along the pulse duration and restrain the voltage drop between the last dynode and the anode, resulting in a significant improvement of the PMT linearity.

To calculate the values of the capacitors, we considered that the PMT has to operate with a gain independent of the anode current. To avoid PMT gain variations with the amount of light received, the ratio between the anode current due to a constant illumination and the current through the voltage divider in absence of illumination has to be less than or equal to 0.01 . $^{10}$

The load resistor $R_{L}$ provides a path to ground for the charge accumulated in the anode in case a fortuitous disconnection of the measuring devices occured. In this way, the charge is unable to damage the equipment when it is reconnecting again.

\section{Light Pulses Transmission}

To excite the PMT with a light source as similar as possible to the Cherenkov light pulses generated in the atmospheric showers, we have used two prototypes of pulsed light transmitters for the tests. The first one is basically an electronic pulse generator based on a Schmidt trigger inverter 74AC14 that drives a high speed LED. This system can provide pulses as short as 10 ns. The second system of light pulsed generator is based on a plastic scintillator excited by a radioactive source, which can provide even shorter pulses a few nanoseconds of duration.

\subsection{Electric Pulse Generator}

The first prototype is shown in Fig. 3. This circuit is built after a simple system of reflectometry in the time domain. ${ }^{11}$ The generator can supply narrow pulses of variable duration, up to $10 \mathrm{~ns}$, and with a repetition frequency also variable, with a minimum value of $10 \mathrm{kHz}$.

The $1 \mathrm{~N} 4148$ device is a high-speed commutation diode with an equilibrium capacitance of $4 \mathrm{pF}$, and it does not need to be polarized. This device controls the commutation time to the ON state, due to the fact that its series resistance in this state is very small compared to $R_{1}$.

When the diode is in the OFF state, the commutation is regulated by $R_{1}$. In this way, the two branches of the feedback allow us to obtain highly asymmetric pulses, which can give in practice working duty cycles up to $0.01 \%$. This value makes it possible to use commercially available LEDs placed at short distances from the PMT without damaging it. Due to this, we could not only characterize the PMT response to very short duration pulses, but also we could make all the measurements inside a box of small dimensions $(1 \times 0.5 \times 0.5 \mathrm{~m})$, which was properly magnetically and electrically shielded. In the box designed for this work, the distance LED to PMT was of only $30 \mathrm{~cm}$, although the LED was polarized to $50 \%$ of its maximum operating voltage during the transmission of the pulse to the LED.

The five parallel HEX inverters act as a buffer, isolating the generator from the charge. Due to the fact that they typically present a $30-\Omega$ output resistance, the $220-\Omega$ series resistors permit good coupling to a $50-\Omega$ charge resistor. 


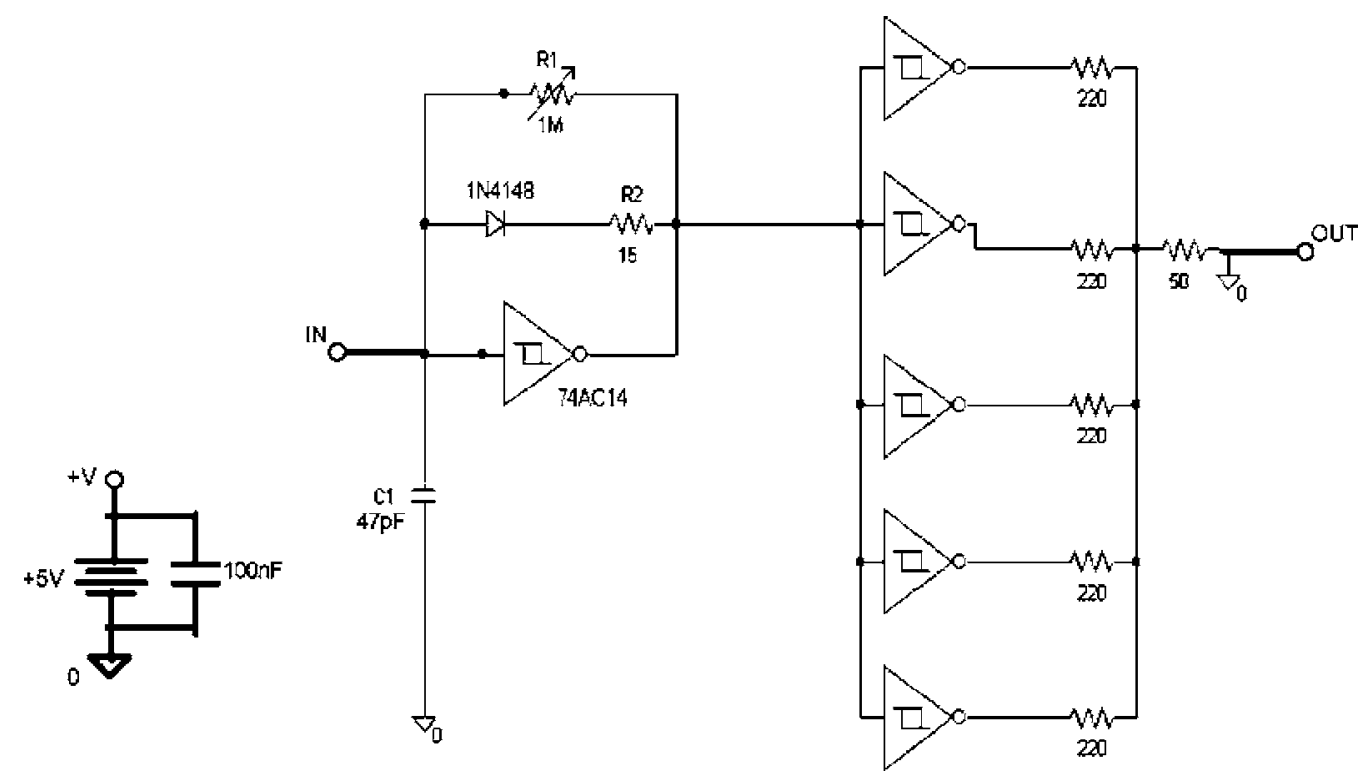

Fig. 3 Pulse generator. The output charge resistor of $50 \Omega$ simulates the oscilloscope used in the tests. The inverters are fed with a 5-V power supply. The 100-nF blocking capacitor enhances the time response of the system as mentioned in the text.

The output impedance can be modified in a simple way by using an additional series-connected resistor to couple to the other charge resistors.

The amount of light due to pulses of the order of nanoseconds is very small; therefore a pulse generator of this type can help to measure the sensitivity of the PMT for very low illumination levels. Using light attenuators, one can even implement the detection of single photons.

The simulation of the generator was made with the program SPICE, using for the model of the Schmidt Trigger inverter values of the rise and fall times given by the manufacturer (Table 1).

The simulations show that the width of the pulse can be controlled by varying the value of the capacitor $C_{1}$ or the value of the resistor $R_{2}$. The repetition frequency of the pulses is controlled with the variable resistor $R_{1}$. With the values shown in Fig. 3, a train of pulses of $10 \mathrm{~ns}$ wide and $20-\mathrm{KHz}$ frequency was obtained.

The stability of the polarization of the inverters was obtained through a blocking capacitor higher than $100 \mathrm{nF}$, which is connected between the power supply and ground. The pulses have important frequency components in the rf bands; therefore, the blocking capacitor should be preferably made of ceramic or mica.

The lack of stability in the voltage supply can affect not only the efficiency but also the width and duration of the pulse as well as the rise time. To quantify these effects, we have made several measurements of the pulses with and without the blocking capacitor. Figure 4(a) shows a com-

Table 1 Rise and fall times of the 74AC14 inverters (ns).

\begin{tabular}{cccc}
\hline \hline Typical $t_{\mathrm{PLH}}$ & Max. $T_{\mathrm{PHL}}$ & Typical $t_{\mathrm{PHL}}$ & Max. $t_{\mathrm{PHL}}$ \\
\hline 7 & 10 & 6 & 8.5 \\
\hline \hline
\end{tabular}
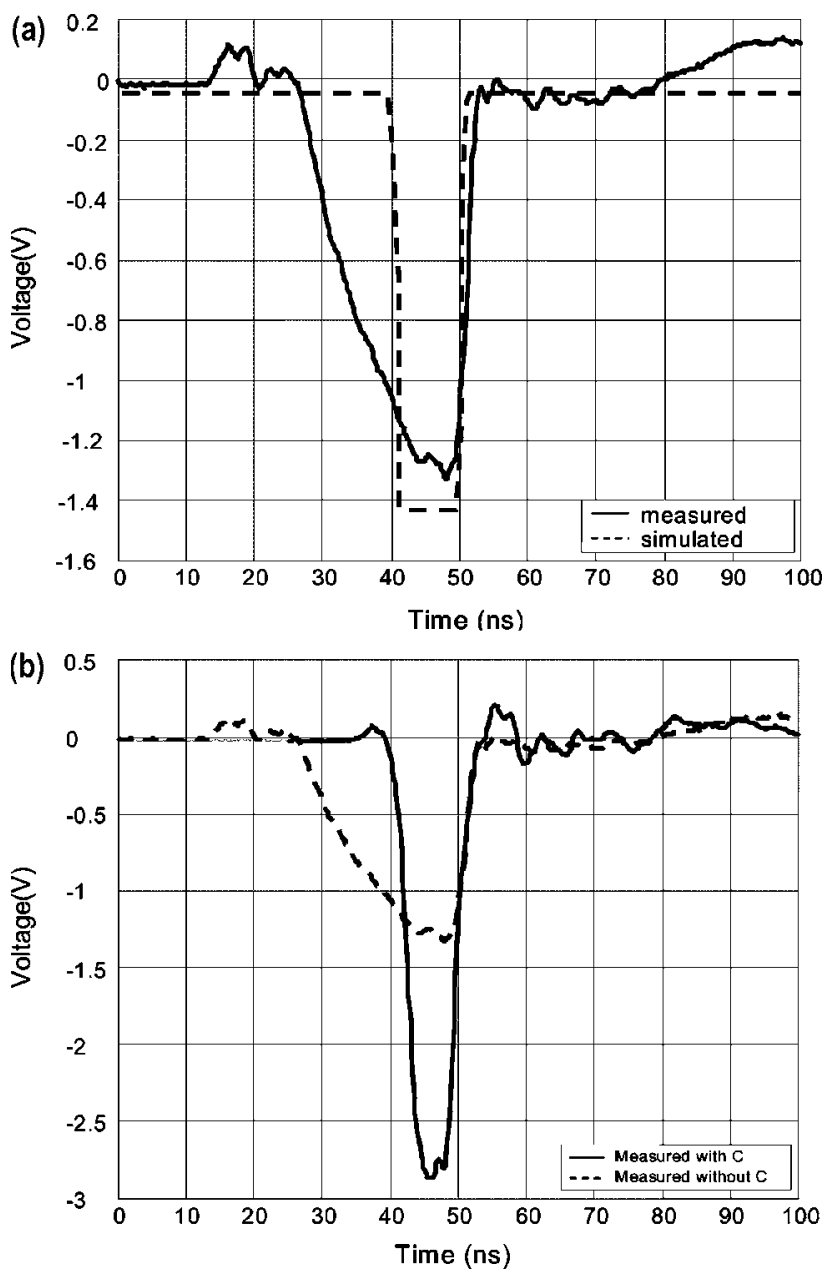

Fig. 4 (a) Measured and simulated pulse without blocking capacitor (C). The rise and fall time given by the manufacturer were used for the simulations. (b) Pulse measured with and without blocking capacitor (C). 

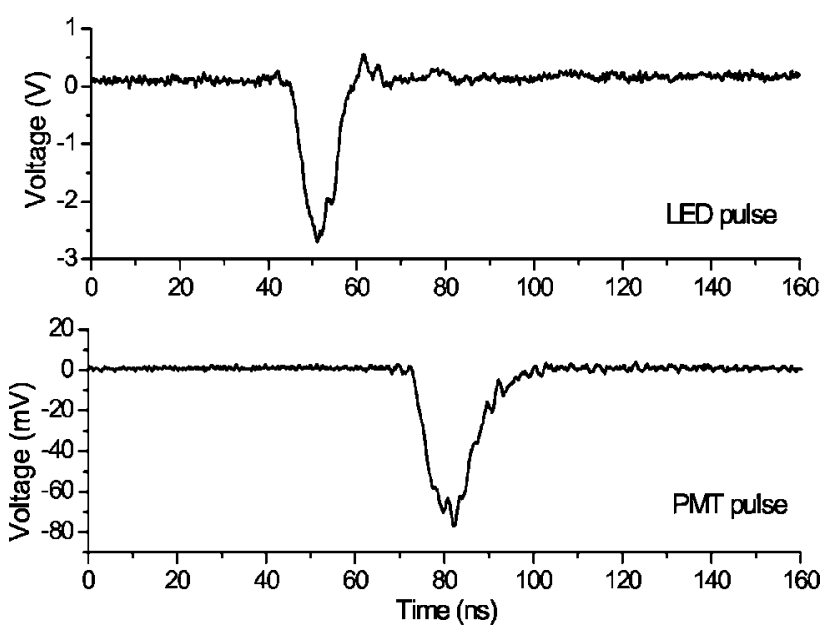

Fig. 5 Comparison between the pulse driving the LED (upper plot) and pulse detected by the PMT (lower plot).

parison between the simulated and measured pulses without the blocking capacitor. The simulation is able to predict the amplitude and the fall time with good accuracy. However, the rise time is significantly degraded, such that the effective duration of the pulse is about two times wider than the theoretical value. Figure 4(b) shows a comparison of the generated pulse as measured with and without the blocking capacitor. A 500-MHz digital phosphorus oscilloscope TDS3052 Tektronix (Wilsonville, Oregon) was used.

The pulse generator was applied to a high speed $\mathrm{GaN}$ LED L7113PBC from Kingbright (City of Industry, California), with a maximum light intensity of $1 \mathrm{~cd}$ for a peak wavelength $\lambda_{\text {LED }}$ of $468 \mathrm{~nm}$. In this way, very short pulses $10 \mathrm{~ns}$ wide and peaked in the UV band as the typical Cherenkov pulses were obtained. An equivalent circuit model for this LED was used to evaluate the possible mismatches between the generator and the device. ${ }^{12}$ The relatively high series resistance of these devices helps to match the generator to the LED, and therefore no coupling networks are needed to avoid pulse distortions.

Figure 5 shows one of the pulses polarizing the LED (forward voltage $V_{f}=2.72 \mathrm{~V}$ ) and the PMT anode pulse in response to the light emitted $\left(\mathrm{V}_{\text {pulse }}=76 \mathrm{mV}\right)$.

It can be seen that the device has a good time response, with no significant distortions in the pulse shape. During the time at which the excitation voltage is positive, as shown in Fig. 5, the LED is in reverse mode and it does not emit; therefore, the weak ripple generated after the pulse is not transmitted to the photomultiplier.

The delay time of the PMT response versus the arrival time of the exciting pulse can be estimated from Fig. 5 . Taking into account that there is a delay of about $15 \mathrm{~ns}$ due to the length of the cable used, we have estimated that the transit time delay of the PMT is about $15 \mathrm{~ns}$, which agrees with the specifications given by the manufacturer. Figure 6 shows the pulse repetition frequency and the corresponding PMT output.

Figure 5 was obtained at a fixed LED polarization of $4 \mathrm{~V}$. By varying the voltage driving the pulse generator, we can obtain different light pulse amplitudes from the LED.
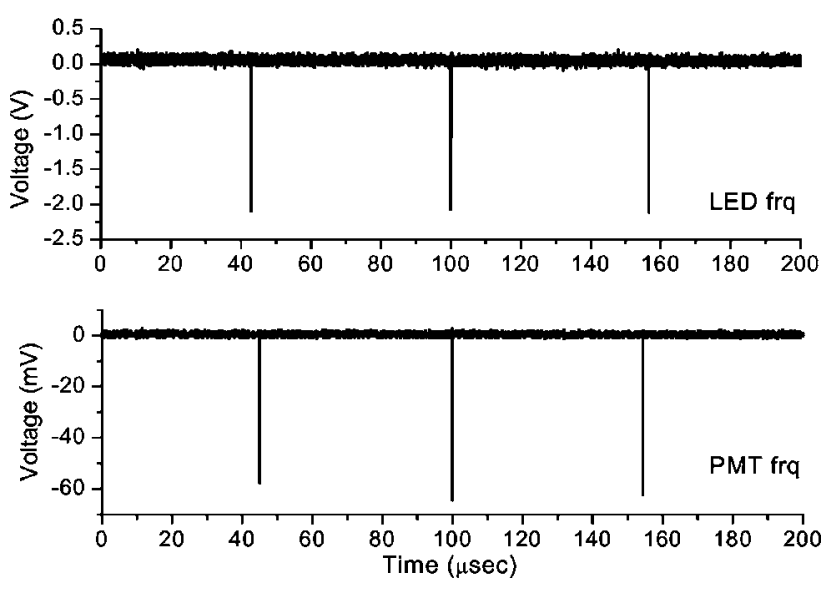

Fig. 6 The pulse train driving the LED (upper plot) $(f=20 \mathrm{kHz})$ and the pulses detected by the PMT (lower plot).

Figure 7 shows the PMT output for different pulse amplitudes. The graph shows the good linearity of the PMT response against the light emission.

\subsubsection{Anode charge estimation}

To estimate the anode current expected for a given pulse amplitude, we have to know the emission spectrum of the LED, the angular distribution of the emitted light, the quantum efficiency (QE) of the PMT as a function of the incident light wavelength, and the PMT gain. The LED emission spectrum and the PMT QE shown in Fig. 8 are usually provided by the manufacturer.

The spatial distribution of the light emitted by the LED (for a given forward current) was directly measured using a PerkinElmer (Wellesley, Massachusetts) VTB8440B IR filtered photodiode placed $35 \mathrm{~cm}$ from the LED and at the same height. The LED was rotated around the axis perpendicular to the plane containing the photodiode and the LED itself and, for a fixed forward voltage, the photodiode voltage was measured. As shown in Fig. 9, the angular distri-

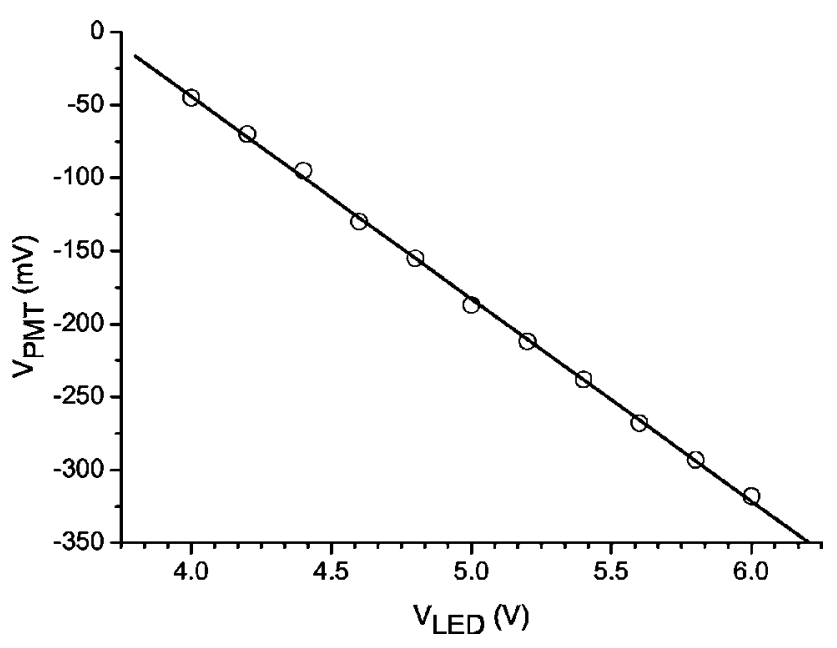

Fig. 7 PMT anode pulse (peak) as a function of the LED polarization. 


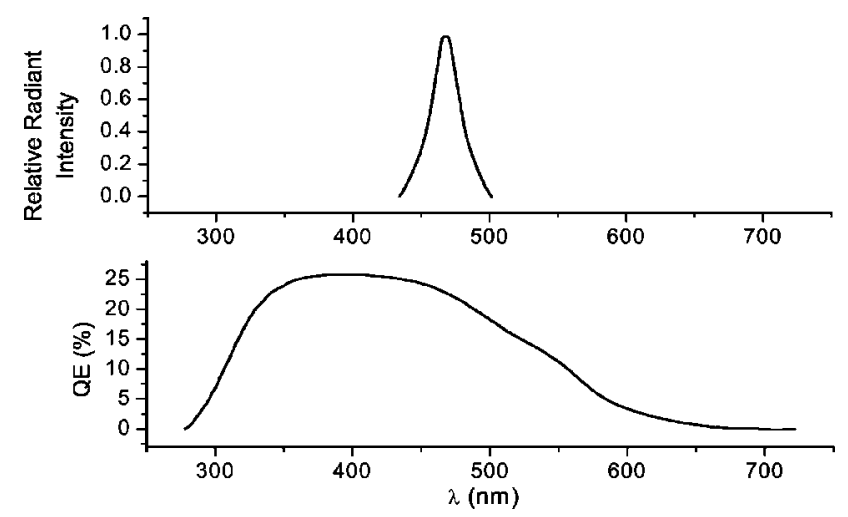

Fig. 8 The relative radiant intensity of the LED L7113 (upper plot) and the spectral response (quantum efficiency) of PMT EMI9116 (lower plot).

bution is not uniform with respect to the LED optical axis, showing a brighter annular ring with a radius of around $8 \mathrm{deg}$.

Knowing these quantities, the number of photoelectrons per second (phe/s) $n$ emitted by the photocatode for a given light intensity will be given by convolving the LED spectrum emission $P(\lambda)$ with the PMT spectral response $Q E(\lambda)$, divided by the photon energy:

$$
\begin{aligned}
n & =\Omega_{\mathrm{LED}} \frac{I_{\text {inc }}}{I_{T}} \int_{\lambda_{i}}^{\lambda_{f}} \frac{P(\lambda) Q E(\lambda)}{h c / \lambda} d \lambda \\
& =\Omega_{\mathrm{LED}} \frac{I_{\text {inc }}}{I_{T}} P\left(\lambda_{\text {peak }}\right) \int_{\lambda_{i}}^{\lambda_{f}} \frac{N(\lambda) Q E(\lambda)}{h c / \lambda} d \lambda[\mathrm{phe} / \mathrm{s}],
\end{aligned}
$$

where $N(\lambda)$ is the relative radiant intensity shown in Fig. 8, $P\left(\lambda_{\text {peak }}\right)$ is the power emitted (in W/sr) for a given forward current $I_{f}, \Omega_{\mathrm{LED}}$ is the solid angle covered by the LED emission, and $I_{\text {inc }} / I_{T}$ is the ratio between the incident light on the detector and the total light emitted by the LED within the solid angle $\Omega_{\mathrm{LED}}$. This ratio takes into account

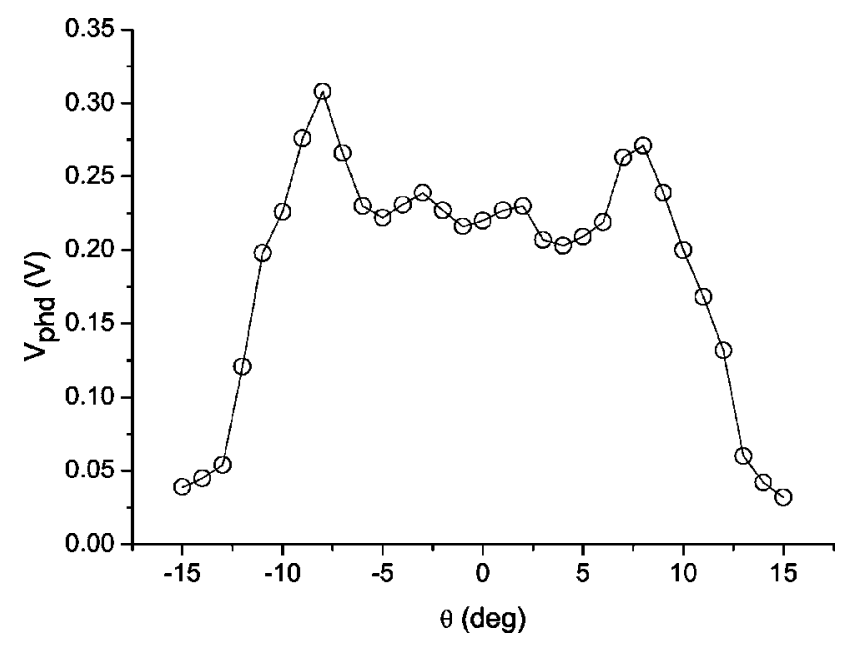

Fig. 9 The angular distribution of the light emitted by the LED Kingbright L7113. $\theta$ is the angle between the LED and photodiode optical axis.

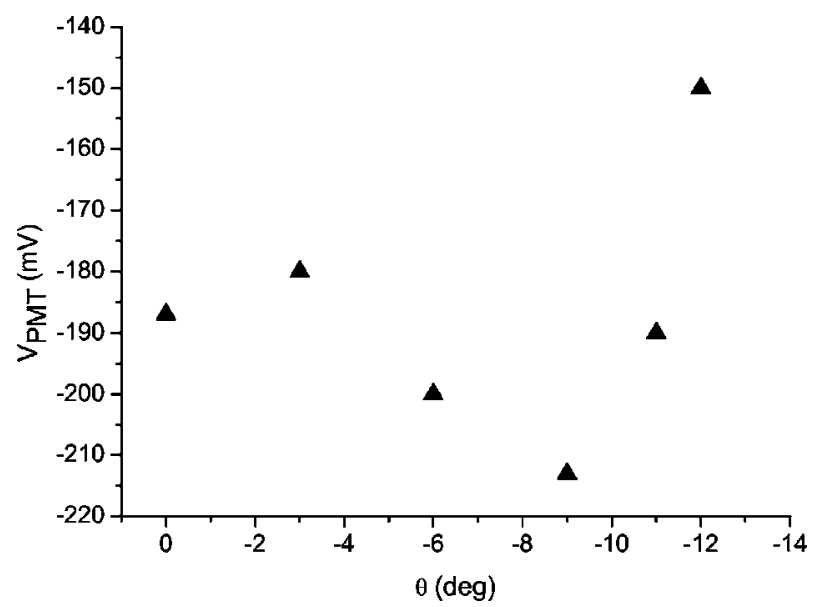

Fig. 10 Voltage at the PMT anode obtained by varying the azimuthal angle of the LED emission.

the solid angle covered by the PMT (and thus, its distance from the LED) and the angular distribution of the emission, and can be substituted by the following expression ${ }^{13}$ :

$\frac{I_{\mathrm{inc}}}{I_{T}}=2 \pi \int_{-\theta_{d}}^{\theta_{d}} F(\theta) \sin (\theta) d \theta$,

where $F(\theta)$ is the angular distribution of Fig. 9 normalized such that its integration over $\Omega_{\mathrm{LED}}$ is 1 . The integration angle $\theta_{d}$ is derived from the detector radius $R$ and the distance $d$ to the LED. In our case, $R=11 \mathrm{~mm}$ and $d$ $=300 \mathrm{~mm}$, thus $\theta_{d}=1.8 \mathrm{deg}$.

For the LED pulse shown in Fig. 5, we have a peak value of $V_{f}=2.72 \mathrm{~V}$. According to the datasheet provided by the manufacturer, this voltage corresponds to a diode current $I_{f}=1.4 \mathrm{~mA}$. This is the $7 \%$ of $20 \mathrm{~mA}$, the current at which the light intensity is known to be $1 \mathrm{~cd}$. Therefore, the emitted power intensity is estimated as $P\left(\lambda_{\text {peak }}\right)=0.07 \mathrm{~cd}$ $\approx 10^{-4} \mathrm{~W} / \mathrm{sr}$.

Substituting in Eq.(1) the curves of Fig. 8 and the ratio $I_{\text {inc }} / I_{T}$ calculated from Fig. 9, we get for the light pulse generated and for the PMT ET9116 placed at $30 \mathrm{~cm}$ from the LED, $n=7.47 \cdot 10^{11} \mathrm{phe} / \mathrm{s}$. Since the PMT has a known gain of $G=10^{4}$, the anode pulse current is estimated to be $I_{a}=1.19 \mathrm{~mA}$. The oscilloscope load impedance was set to $50 \Omega$ in the measurements, and therefore this current leads to a pulse voltage of $V_{\text {pulse }} \approx 60 \mathrm{mV}$. This calculation is in reasonable agreement with the measured value of $76 \mathrm{mV}$. The underestimation of the measurement is attributed to an underestimation of the power radiated by the LED used in the tests. This factor could be accurately measured with a calibrated source, unavailable to the authors at the time of this publication.

Figure 10 shows the variations of the PMT anode charge obtained by rotating the LED in the azimuthal direction. Again, the PMT detects the nonuniformity in the LED angular emission.

\subsubsection{Pulse Generation with a Plastic Scintillator}

To test the response of the polarization network designed for the PMT EMI9116 for pulses shorter than $10 \mathrm{~ns}$, we 


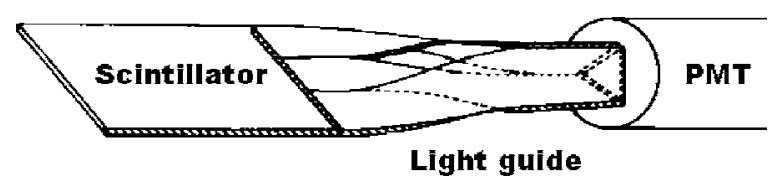

Fig. 11 The coupling scintillator PMT.

used a second prototype as a light pulse transmitter. This second prototype makes use of a plastic scintillator excited by a radioactive source. However, in this case, we cannot modify the frequency, amplitude, and duration of the pulses as in the first prototype.

A scintillator is made of a luminescence material, which emits a small amount of light by fluorescence or phosphorescence when it is crossed by a charged particle, or a gamma photon which ionizes the material. ${ }^{3}$ The emitted light is guided to the PMT, which is coupled to the scintillator by means of a light guide, as shown in Fig. 11. The PMT converts the incident light into an electrical signal that can be analyzed later.

The shape and duration of the scintillator signal gives additional information about the nature of the particles crossing it and, for this reason, the system scintillator PMT is usually used as a radioactivity detector in nuclear and particle physics. First, the intensity of the emitted light is directly proportional to the energy of the ionizing particle going through. Second, the scintillator is very fast and, therefore, one can study with high accuracy the frequency of the phenomena. Finally, when analyzing the pulse shape at the PMT output, one can determine the particle type that goes through the scintillator detector.

The type of material used depends on the type of measurement and radiation to be detected. In our tests, a plastic scintillator has been used to produce pulses of short duration, since the re-emission time of the light impinging on this material is of the order of nanoseconds.

A sample of thorium of low radioactivity placed on the scintillator has been used to excite the material. The radioactive thorium sample decays via emission of $\alpha, \beta$, and $\gamma$ particles. $^{3}$

Figure 12 shows one of the charge pulses detected at the PMT anode, using the polarization given in the scheme of Fig. 2 and with the PMT coupled to a plastic scintillator excited by the radioactive source. As it can be seen, the pulse width is of the order of $5 \mathrm{~ns}$, which proves the capability of the polarization network built to detect such short pulses.

\section{Conclusions}

In this work, a testbench for the characterization of the photomultipliers used in the camera of the MAGIC telescope (EMI 9116) is set up. This testbench incorporates a polarization network for the PMT optimized for operation in pulsed mode and two systems of light pulse generation. The first system is based on a Schmidt Trigger inverter, which drives a high speed UV LED, model Kingbright L7113. The use of blocking capacitors to stabilize the polarization turns out to be critical for the optimization of the PMT linearity and for the duration and rise time of the pulses. With this compact and inexpensive system, very short pulses (down to $10 \mathrm{~ns}$ ) with very small duty cycles

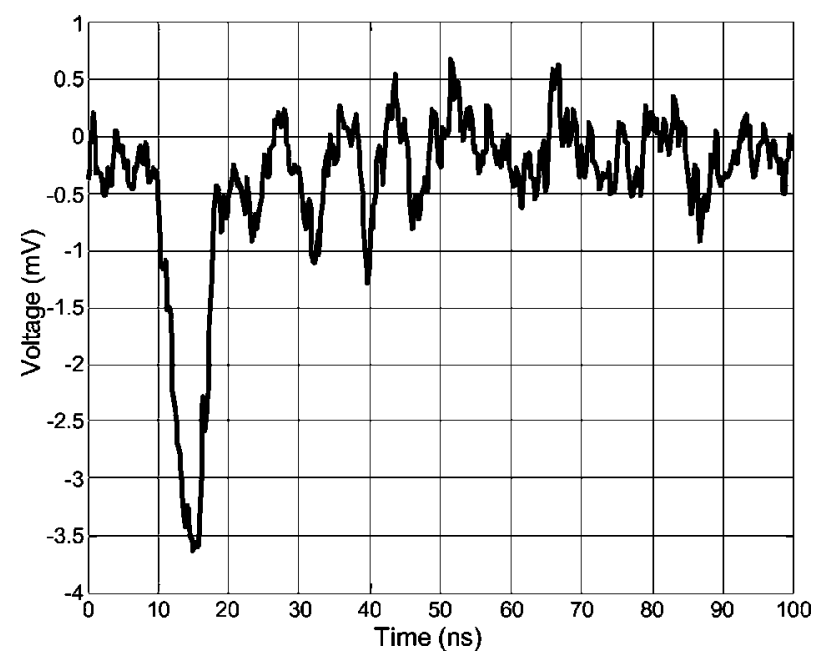

Fig. 12 PMT anode pulse using as a pulse generator a plastic scintillator excited by a radioactive source.

$(0.03 \%)$ are obtained, and this resembles the standard operating conditions of the PMTs mounted in the telescope camera.

From the measurements obtained by coupling this light pulser to the PMT, we find the following results: 1 . the PMT biased with the adopted polarization network can detect 10 -ns pulses without significant distortions; 2 . the measurements allow us to estimate the transit time delay of the PMT in $15 \mathrm{~ns}$, which is significantly longer than the detected pulse; 3 . the use of pulse trains with $0.03 \%$ duty cycle allow us to illuminate the PMT with a strong light intensity without risk of damage. Thus, the light pulser can be located a short distance from the PMT, and therefore the whole testbench can be mounted in a compact assembly that facilitates both magnetostatic and electromagnetic screening.

Along with these measurements, an estimate of the PMT anode charge for a given light intensity is made. The estimation is based on a detailed calculation where the spectrum emission of the LED used for the tests and the PMT spectral response are both considered. The angular distribution of the LED luminosity, which strongly affects the final estimation, is also measured, and the result obtained for the given LED pulse amplitude is comparable with the measured value. The unavailability of a calibrated light source for the determination of the LED radiative power reduces the precision of the estimation. Nevertheless, the number obtained agrees within the errors with the experimental value.

The whole setup has also been used to test a modified version of the MAGIC PMT, which has been recently installed at the center of the MAGIC camera. ${ }^{8,9}$

Using the second system of light pulse generation, composed by a plastic scintillator excited by a radioactive source, we verify that the PMT with the designed polarization network is able to detect very weak signals of duration shorter than $10 \mathrm{~ns}$.

In the near future, we plan to use the electro-optical testbench for measurements with a so-called hybrid 
photomultiplier, ${ }^{14}$ a novel type of photosensor that replaces the multiplying unit (dynodes) of a photomultiplier by a single avalanche photodiode.

\section{Acknowledgments}

The authors thank the financial support given by the CICYT (project FPA2003-9543-C02-01). We also acknowledge Eckart Lorenz from the Max-Planck-Institut für Physik in Munich for his valuable help.

\section{References}

1. M. V. Fonseca, "The MAGIC telescope project," Acta Polonica B 30, 2331 (1999)

2. T. K. Gaisser, "Cosmic rays and particle physics," Cambridge University Press, Boston, MA (1990).

3. W. R. Leo, Techniques for Nuclear and Particle Physics Experiments, 2nd ed., Springer-Verlag, Berlin (1987).

4. A. Ostankov, D. Paneque et al., "A study of the new hemispherical 6-dynodes PMT from electron tubes," Nucl. Instrum. Methods Phys. Res. A 442(1-3), 117-123 (2000).

5. J. Gebauer et al., "Evaluation of a new high QE photomultiplier for air Cherenkov telescopes," Nucl. Instrum. Methods Phys. Res. A 518, 615 (2004)

6. E. Lorenz et al., "Progress on the development of a high QE, red extended hybrid photomultiplier for the 2nd phase of the MAGIC telescope," Nucl. Instrum. Methods Phys. Res. A 504, 280-285 (2003).

7. P. Antoranz, F. Lucarelli, M. Asensio, J. M. Miranda, and M. V. Fonesca, "A simple blue light pulse generator with $\mathrm{GaN} / \mathrm{SiC}$ light emitting diodes for the time response testing of PMTs," Proc. SPIE 5840, 192-200 (2005).

8. M. Asensio, F. Lucarelli, P. Antoranz, J. A. Barrio, J. M. Miranda, and M. V. Fonesca, "Design, modelling, and testing of electro-optical transmitters for the central pixel of the MAGIC telescope camera,"
Proc. SPIE 5840, 627-637 (2005).

9. F. Lucarelli et al., "Development and first results of the MAGIC central pixel system for optical observations," Proc. 29th Intl. Cosmic Ray Conf., pp. 367-370 (2005).

10. S. O. Flyckt and C. Marmonier, "Photomultiplier tubes: Principles and applications," Photonis, 5-2 (2002).

11. T. Engdahl, see http://www.hut.fi/Misc/Electronics/circuits/tdr.html.

12. P. Antoranz, J. M. Miranda, and J. L. Sebastián, "High frequency modeling of $\mathrm{GaN} / \mathrm{SiC}$ blue light emitting diodes," J. Appl. Phys. 97, 096107 (2005).

13. M. Cámara, F. Lucarelli, M. V. Fonseca, I. Oya, P. Antoranz, and J. M. Miranda, "An analytical model of a LED-based transmission system for the test of highly sensitive photodetectors" (in progress).

14. Compact HPD (hybrid photo-detector), see http://www.hpk.co.jp/ Eng/products/ETD/hpde/hpde.htm.

Fabrizio Lucarelli graduated in physics at La Sapienza University of Rome, Italy, in the branch of astrophysics. He earned his doctorate in physics at the Complutense University of Madrid, Spain, with a thesis entitled "Observation of the Monoceros Loop SNR with the HEGRA system of Cherenkov telescopes." $\mathrm{He}$ is an expert in the field of astroparticle physics, in particular TeV gamma-ray astronomy by ground-based detectors and neutrino astronomy. $\mathrm{He}$ specializes in the different aspects of the exploitation and performance of very high energy gamma ray $(50 \mathrm{GeV}$ to $50 \mathrm{TeV})$ detectors using the imaging air Cherenkov telescope technique. He was a member of the High Energy Gamma Ray Astronomy (HEGRA) collaboration. $\mathrm{He}$ is the author of three refereed papers and coauthor of more than 25 papers with the HEGRA and MAGIC collaboration. Presently, he has a tenure track at the Rome University La Sapienza, where he is teaching physics and continuing his research in the field of gamma-ray and neutrino astronomy.

Biographies of other authors not available. 